\title{
Laser safety in fiber-optic monitoring of spinal cord hemodynamics: a preclinical evaluation
}

David R. Busch

James Davis

Angela Kogler

Robert M. Galler

Ashwin B. Parthasarathy

Arjun G. Yodh

Thomas F. Floyd 


\title{
Laser safety in fiber-optic monitoring of spinal cord hemodynamics: a preclinical evaluation
}

\author{
David R. Busch, ${ }^{\mathrm{a}, \mathrm{b}, \mathrm{c}, \star}$ James Davis, ${ }^{\mathrm{d}}$ Angela Kogler, ${ }^{\mathrm{e}, \mathrm{f}}$ Robert M. Galler, ${ }^{\mathrm{g}}$ Ashwin B. Parthasarathy, ${ }^{\mathrm{h}}$ \\ Arjun G. Yodh, ${ }^{\mathrm{c}}$ and Thomas F. Floyd ${ }^{\mathrm{a}, \star}$ \\ anniversity of Texas Southwestern, Department of Anesthesiology and Pain Management, Dallas Texas, United States \\ bUniversity of Texas Southwestern, Department of Neurology and Neurotherapeutics, Dallas, Texas, United States \\ 'University of Pennsylvania, Department of Physics and Astronomy, Philadelphia, Pennsylvania, United States \\ dStony Brook University Medical Center, Department of Pathology, Stony Brook, New York, United States \\ 'Stony Brook University Medical Center, Department of Anesthesiology, Stony Brook, New York, United States \\ 'Stony Brook University, Department of Biomedical Engineering, Stony Brook, New York, United States \\ 'Stony Brook University Medical Center, Department of Neurosurgery, Stony Brook, New York, United States \\ hUniversity of South Florida, Department of Electrical Engineering, Tampa, Florida, United States
}

\begin{abstract}
The prevention and treatment of spinal cord injury are focused upon the maintenance of spinal cord blood flow, yet no technology exists to monitor spinal cord ischemia. We recently demonstrated continuous monitoring of spinal cord ischemia with diffuse correlation and optical spectroscopies using an optical probe. Prior to clinical translation of this technology, it is critically important to demonstrate the safety profile of spinal cord exposure to the required light. To our knowledge, this is the first report of in situ safety testing of such a monitor. We expose the spinal cord to laser light utilizing a custom fiber-optic epidural probe in a survival surgery model (11 adult Dorset sheep). We compare the tissue illumination from our instrument with the American National Standards Institute maximum permissible exposures. We experimentally evaluate neurological and pathological outcomes of the irradiated sheep associated with prolonged exposure to the laser source and evaluate heating in ex vivo spinal cord samples. Spinal cord tissue was exposed to light levels at $\sim 18 \times$ the maximum permissible exposure for the eye and $\sim(1 / 3) \times$ for the skin. Multidisciplinary testing revealed no functional neurological sequelae, histopathologic evidence of laser-related injury to the spinal cord, or significant temperature changes in ex vivo samples. Low tissue irradiance and the lack of neurological, pathological, and temperature changes upon prolonged exposure to the laser source offer evidence that spinal cord tissues can be monitored safely with near-infrared optical probes placed within the epidural space. @ 2018 Society of Photo-Optical Instrumentation Engineers (SPIE) [DOI: 10.1117/1.JBO.23.6.065003]
\end{abstract}

Keywords: spinal cord; ischemia; optical monitoring; hemodynamics; diffuse optics; intraoperative monitoring.

Paper 180118RR received Feb. 23, 2018; accepted for publication May 30, 2018; published online Jun. 19, 2018.

\section{Introduction}

Spinal cord injuries can occur as a result of disease (e.g., tumors), trauma (e.g., accidents), or complications from surgeries, such as scoliosis correction, ${ }^{1}$ spinal cord tumor resection, ${ }^{2}$ and aortic aneurysm repair. ${ }^{3,4}$ The prevention and treatment of spinal cord injury are focused upon the maintenance and/or restoration of spinal cord blood flow, yet no technology exists to monitor spinal cord ischemia. This is a critical gap in the field of neurocritical care monitoring. Recently, we developed and demonstrated continuous monitoring for spinal cord ischemia using a thin, flexible fiber-optic probe employing near-infrared (NIR) technology. ${ }^{5,6}$ Furthermore, testing is needed prior to clinical adoption of this concept, including a clear demonstration that deployment of the required laser light in the proximity of the spinal cord is safe. This type of safety study is especially important because unlike many tissues in the body, even minimal damage to spinal tissues, as might arise in a "burn," can lead to paralysis or paraparesis.

\footnotetext{
*Address all correspondence to: David R. Busch, E-mail: david.busch@ utsouthwestern.edu; Thomas F. Floyd, E-mail: thomas.floyd@utsouthwestern .edu
}

Current methods available for assessment of spinal cord ischemia during surgery are indirect. Generally, they are based upon neuro-electrophysiological principles, especially somatosensory and motor-evoked potentials (SSEP and MEP, respectively), which monitor the integrity of posterior spinal sensory pathways and the anterior/lateral spinal motor tracts, respectively. When combined, these modalities can help to identify injury and can offer the surgeon insight into the impact of interventions and opportunities to limit or reverse injury., ${ }^{7,8}$ Interpretation of these data, however, requires the presence of a neurologist skilled in neuro-electrophysiological monitoring. This monitoring, in turn, may be influenced by anesthetics,, 10 patient temperature, ${ }^{11}$ ischemia (cord and limb), and mechanical mechanisms. "False negatives," wherein patients awaken with important deficits in spite of "normal" evoked potentials, as well as "false positives," wherein patients awaken without deficits in spite of loss or degradation of signal, have been reported with both $\mathrm{SSEP}^{12}$ and $\mathrm{MEP}^{13}$ monitoring, even when used in a complimentary fashion. ${ }^{14}$

Further, neuro-electrophysiological alerts may be temporally insensitive (delayed) relative to the inciting event, ${ }^{15,16}$ which

$1083-3668 / 2018 / \$ 25.00$ (C) 2018 SPIE 
diminishes the chance for rescue of threatened tissue. To complicate the situation more, recovery of signals after rescue attempts is also markedly delayed, leaving the surgeon in a quandary per how to proceed. MEPs require application of high currents to the scalp, causing significant pain, and can thus only be performed in heavily sedated patients. SSEPs can be performed in awaken patients, but the electrical stimulation is still painful and current spinal cord monitoring modalities cannot monitor patients continuously in ICU settings. Finally, these techniques are not specific. A 2012 evidencebased clinical guideline noted that only $16 \%$ to $40 \%$ patients with significant intraoperative electrophysiological changes developed postoperative-onset paraparesis, paraplegia, or quadriplegia. ${ }^{17}$ Nevertheless, despite the well-documented deficiencies of this methodology, MEP and SSEP are still considered the "gold standard" for functional monitoring of the spinal cord for spine, spinal cord, and aortic surgery. A relatively new monitor utilizes the perfusion pressure to the spinal column to identify ischemic risk, ${ }^{18,19}$ yet this is still an indirect measurement of ischemia.

Recently, we demonstrated and validated direct monitoring of spinal cord hemodynamics in sheep using a custom fiberoptic probe and optical monitor based on diffuse optical spectroscopy and diffuse correlation spectroscopy (DOS and DCS, respectively) ${ }^{5,6}$ DCS is a relatively new technology capable of measuring microvascular blood flow. We have previously validated it against fluorescent microsphere measurements of spinal cord blood flow in a sheep model. ${ }^{6} \mathrm{We}$, and others, have validated this technology in brain and muscle against microspheres, MRI, Xe-CT, and Doppler ultrasound. ${ }^{20-26}$ DCS and DOS employ NIR light to probe tissues up to a few centimeters below the surface with high temporal resolution $(>0.5 \mathrm{~Hz})$. These instruments are minimally invasive, portable, ${ }^{27-30}$ and they have been successfully employed to measure hemodynamics in a variety of tissues, including the brain, ${ }^{21,31-34}$ bone, ${ }^{35,36}$ cancer, ${ }^{37,38}$ and muscle, ${ }^{39,40}$ both at the bedside and intraoperatively. ${ }^{41-43}$ However, while optical techniques have been used for continuous long-term monitoring of tissues, ${ }^{22}$ such measurements typically employ low optical powers/fluence rates, and often, the input light travels through barrier tissues (e.g., scalp/skull), which effectively protect more sensitive tissues (e.g., cerebral cortex) from injury due to light radiation by reducing light energy deposition.

The importance of demonstrating the safety of optical monitoring techniques for the spinal cord cannot be overstated: injury to spinal cord tissues can result in life-changing paralysis or paraparesis. By comparison to other tissues, skin burns or even localized retinal damage have relatively minor impact on patients' quality of life. Spinal surgeries already carry some degree of risk; a new monitoring device cannot add significantly to this burden. Despite this need and the rapidly advancing use of related optical tools in the clinic, we are not aware of any published reports focused on optical radiation safety in long-term monitoring of the spinal cord, and current optical safety standards do not address this application. ${ }^{44,45}$

A few papers have focused on therapeutic applications of lasers near the spinal cord, e.g., for laser disc decompression. These therapies frequently utilize longer wavelengths (e.g., $1064 \mathrm{~nm}$ ) and higher power to deliberately heat or ablate tissue in a single brief session. A report of 2400 percutaneous laser disc decompressions involving tissue ablation found no nerve or spinal cord damage, ${ }^{46}$ although damage to the nerve roots was reported in another study. ${ }^{47}$ There have also been recent suggestions that light of similar wavelengths may be utilized to enhance healing of the spinal cord. ${ }^{48,49}$

Our previous spinal cord work ${ }^{5,6}$ focused on proof-of-concept demonstrations of optical monitoring of the spinal cord but did not address the safety of these measurements as pertains to optical spinal cord injury. Indeed, laser-related safety for long-term monitoring with diffuse light has never been tested in the spinal cord and these safety questions should be considered before moving these monitoring technologies to the spinal cords of human subjects. As discussed above, injury to the spinal cord is significantly more life-altering than damage e.g., to the skin. Thus, the current American National Standards Institute (ANSI) standard, ${ }^{44}$ which is focused on skin and ocular exposure, ${ }^{45}$ lacks the specificity needed for the optical spine monitoring problem and, thus, speaks directly to the gap in scientific knowledge in this niche and to the importance of a safety study.

Given this knowledge gap and the serious consequences of spinal cord damage, it is important to directly and explicitly demonstrate the safety of optical monitoring of the spinal cord prior to undertaking human studies. In an animal model, the geometry and thermal dissipation are the key factors to replicate, e.g., the spine of a small animal model would have a significantly different heat capacity. To this end, we explore the safety issue using a large animal model (sheep), which closely approximates the geometry, ${ }^{50}$ thermal dissipation, and vulnerability to injury of the human spine and spinal cord. Specifically, we carry out monitoring measurements on sheep spinal cord with DCS and DOS laser illumination. We compare the tissue illumination from our instrument with ANSI maximum permissible exposures (MPE), and we evaluate neurological and pathological outcomes of the irradiated sheep with prolonged exposure to the laser source. Finally, tissue heating is evaluated in ex vivo spinal cord samples.

\section{Materials and Methods}

Experiments were conducted in accordance with the Institutional Animal Care and Use Committee (Stony Brook University, Stony Brook, New York) guidelines.

\subsection{Diffuse Optical Opto-Electronics}

A "hybrid" optical system was utilized in this study, comprising of a commercial DOS system (Imagent, ISS Inc., Urbana, Illinois) and a lab-built DCS system, which has been described previously. ${ }^{6}$ Briefly, DOS relates the wavelength-dependent differential absorption of light transmitted through tissue to a quantitative diffusion model of light propagation and estimates the baseline/absolute concentrations of tissue chromophores, such as oxy- and deoxy-hemoglobin. ${ }^{27}$ DCS measures the relative blood flow in deep tissues by quantifying the temporal intensity fluctuations of the detected light, which occur mainly due to the motion of red blood cells. ${ }^{29,51,52}$ Blood flow is estimated from the decay rate of a temporal intensity autocorrelation function, which quantifies the light intensity fluctuations. We direct the interested reader to recent review articles ${ }^{27,53}$ for more details about these technologies, including their mathematical underpinnings and an online summary of papers on DCS applications. ${ }^{26}$

The custom DCS module employs a long coherence length 785-nm laser diode (CrystaLaser Inc., Nevada, DL785-100$\mathrm{SO})$ with nominal operating power of $100 \mathrm{~mW}$. Light is detected 


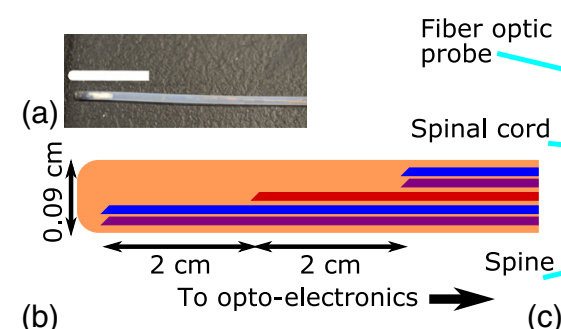

(b)

\begin{abstract}
Fig. 1 (a) Photograph of distal tip of probe (1-cm scale bar, white). (b) Diagram of distal tip of probe (not to scale) showing detector (DCS: blue and DOS: purple) and shared source (red) fibers in a protective Teflon sheath. (c) Photograph of probe in place and exposed spinal cord at the end of the experiment after spinal resection (2-cm scale bar, white). (d) Timeline of optical data frame with relative laser power (to scale). Note that the irradiance from the DCS laser is much higher than the DOS lasers, and thus, the DCS laser dominates the laser safety profile of the hybrid device.
\end{abstract}

using single-mode optical fibers coupled to avalanche photodiode detectors (SPCM-AQ4C, Excelitas), and the photon correlation functions are computed using a customized (correlator.com) USB autocorrelator board.

The commercial DOS system (Imagent, ISS, Illinois) utilizes laser diodes at 686,751 , and $831 \mathrm{~nm}$ modulated at $110 \mathrm{MHz}$, with average operating powers of $2.7,4$, and $4 \mathrm{~mW}$, respectively. This frequency-domain system quantifies tissue scattering and absorption thereby facilitating the calculation of absolute concentrations of oxygenated and deoxygenated hemoglobin. None of the wavelengths utilized have strong wavelength dependency in standards for optical power limits; all lasers utilized have a wavelength error and bandwidth of $<2 \mathrm{~nm}$. Note that the DCS technique has an inherently low signal-to-noise ratio, requiring much higher input power (27 versus $\leq 4 \mathrm{~mW}$ ) and integration time $[2500$ versus $30 \mathrm{~ms}, 35 \%$ versus $0.4 \%$ duty cycle, shown schematically in Fig. 1(d)]. In this configuration, the energy deposited in tissue is, therefore, dominated by the DCS component. The ANSI standard also provides for consideration of energy deposition per pulse; only the DCS laser exposure came even to a few percent of this limit.

\subsection{Fiber-Optic Probe}

Conventionally, illumination and detection of light are realized using flexible fiber-optic cables. For the minimally invasive measurements, our fiber-optic probe (Fiberoptic Systems Inc., Simi Valley, California) is comprised of one laser source fiber (IR100/110/125P, 100- $\mu \mathrm{m}$ core, $0.22 \mathrm{NA}$ ), with DOS (IR100/110/125P, 100- $\mu \mathrm{m}$ core, 0.22 NA) and DCS (Cu800 6/125/165, 0.13 NA, IVG Fiber, Toronto, Ontario, Canada) detector fibers located $2 \mathrm{~cm}$ axially from the source; all fibers are side-firing (see Fig. 1). These fibers were chosen to provide the best compromise between core size and total fiber size inside the 0.9 -mm-diameter probe.

\subsection{Surgical, Anesthetic, and Experimental Procedures}

Eleven adult Dorset sheep, approximately 2 years of age and weighing 60 to $70 \mathrm{~kg}$, were used for the neurological and histological evaluations. Sheep are the preferred animal model for these experiments as ovine spinal anatomy closely models that of humans (e.g., spinal canal depth and width). ${ }^{50}$ Animals were pretreated with glycopyrrolate $(0.02 \mathrm{mg} / \mathrm{kg}, \mathrm{IM})$. Anesthesia was induced with ketamine 10 to $20 \mathrm{mg} / \mathrm{kg}$ IM, animals were intubated, and ventilation controlled. Anesthesia was maintained with isoflurane (1.5\% to $3.0 \%)$. Blood pressure, electrocardiogram, ventilation rate, tidal volume, end-tidal $\mathrm{CO}_{2}$, and arterial oxygen saturation (pulse oximetry) were continuously monitored throughout the procedure.

After adequate general anesthesia was established, the sheep was positioned prone. To test two commonly used clinical surgical procedures, the fiber-optic probe was placed via laminotomy ( $N=3$, "open") and via percutaneous ( $N=8$, "closed") approaches into the epidural space. The percutaneous approach carries a greater risk of spinal cord trauma related to the needle employed, whereas the open approach virtually eliminates this risk of mechanical injury.

To create the laminotomy, a midline incision was made and a subperiosteal dissection was performed to expose the spinous processes, lamina, and medial facets. A Leksell rongeur was then used to remove the intraspinous ligaments as well as the spinous processes themselves. A thin-footed Kerrison rongeur was used to carefully remove the lamina creating a trough that was sequentially widened until the dura of the spinal cord was well seen. The probe was placed using loupe magnification and

Table 1 Summary of the duration and technique of fiber-optic probe placement, including the vertebral level at which probe was introduced and the final location of the probe tip on the spinal cord.

\begin{tabular}{lcccc}
\hline Sheep \# & Duration (h) & Technique & $\begin{array}{c}\text { Probe entry } \\
\text { level }\end{array}$ & $\begin{array}{c}\text { Probe } \\
\text { tip level }\end{array}$ \\
\hline 1 & 4 & Laminotomy & L4 & L2 \\
2 & 4 & Laminotomy & L5 & L2 \\
3 & 4 & Laminotomy & L3 & L1 \\
4 & 4 & Percutaneous & L1 & T13 \\
5 & 4 & Percutaneous & T14 & T12 \\
6 & 4 & Percutaneous & L6 & L4 \\
7 & 6 & Percutaneous & L6 & L4 \\
8 & 6 & Percutaneous & L6 & L3 \\
9 & 6 & Percutaneous & L6 & L2 \\
10 & 6 & Percutaneous & L7 & L2 \\
11 & 6 & Percutaneous & L8 & L3 \\
\hline
\end{tabular}


was advanced superiorly or inferiorly under intact laminae to lie within the epidural space. Percutaneous placement of the probe was achieved via 17 gauge Tuohy needle introduced a lumbar interspace, with the epidural space identified by loss of resistance to air or saline. The probe was then advanced to the desired level under fluoroscopic guidance.

Table 1 summarizes the placement technique (laminectomy or percutaneous), vertebral level at which the probe was introduced, its final location on the spinal cord (confirmed fluoroscopically for percutaneous approaches), and study duration. After introduction into the epidural space, signal was optimized by rotation and axial adjustment along the spine. The probe was then left in place for 4 or $6 \mathrm{~h}$, without being moved. No other interventions were performed during this period.

\subsection{Measurement of Light Input to Tissue}

In considerations of tissue safety, it is the light input to tissue that is of concern rather than the rated power of the sources, i.e., the distal fiber output after taking into account all coupling and transmission losses. Table 2 summarizes the optical powers from the lasers at the distal end of the source fiber (the light source; all lasers were coupled into a single-source fiber). Also, the duration of laser light exposure ( $t_{\text {exp }}$ ) of each light source (laser) is indicated. Note that all of this light exposure is typically contained within a single data collection "frame" (a single DOS and DCS data point) of $\sim 7.25 \mathrm{~s}$. Data might typically be collected in a clinical environment throughout a period of 4 to $6 \mathrm{~h}$, with a duty cycle between 5\% and 35\%. Here, the DCS laser illuminated the tissue with the highest average power (27 versus $\sim 3$ to $4 \mathrm{~mW}$ ) and duty cycle (35\% versus $0.4 \%$ ); both parameters mimic our current experimental protocol.

\subsection{Calculation of Maximum Permissible Exposures}

The ANSI (Z136.1, 2014) ${ }^{45}$ defines a wavelength-dependent MPE. To apply this standard, we note that the laser wavelengths of the DCS and DOS sources are in the range of 680 to $840 \mathrm{~nm}$ (Table 2). In this case, for a point source with wavelengths between 500 and $1050 \mathrm{~nm}$ and exposure durations from 10 to $30,000 \mathrm{~s}$, the MPE (irradiance in $\mathrm{mW} / \mathrm{cm}^{2}$ ) for skin is

$$
\mathrm{MPE}_{\text {skin }}=0.2 C_{A} \text {. }
$$

The corresponding MPE for ocular exposure is

$\mathrm{MPE}_{\text {eye }}=0.001 C_{A}$,

Table 2 Summary of the optical powers at tissue surface.

\begin{tabular}{lccc}
\hline & $\lambda(\mathrm{nm})$ & $P(\mathrm{~mW})$ & $t_{\exp }(\mathrm{ms})$ \\
\hline DOS & 686 & 2.7 & 30 \\
DOS & 751 & 4 & 30 \\
DOS & 831 & 4 & 30 \\
DCS & 786 & 27 & 2500 \\
\hline
\end{tabular}

Note: Optical power $(P)$, laser wavelength $(\lambda)$, tissue exposure duration $\left(t_{\text {exp }}\right)$ of the laser source during a $\sim 7.25$-s data frame, for diffuse optical spectroscopy (DOS), and diffuse correlation spectroscopy (DCS). where $C_{A}$ is a wavelength-dependent factor $\left(\mathrm{mW} / \mathrm{cm}^{2}\right)$, i.e.,

$$
\begin{aligned}
& C_{A}(500<\lambda<700 \mathrm{~nm})=1, \\
& C_{A}(700 \leq \lambda<1050 \mathrm{~nm})=10^{2 \frac{\lambda-700}{1000}},
\end{aligned}
$$

where $\lambda$ is the illumination wavelength in $\mathrm{nm}$. For fiber-optic illumination with small cores (e.g., here, with $\sim 100-\mu$ m core diameters), ANSI Z136.1 establishes that illumination areas $\left(A_{\text {lim }}\right)$ be calculated based on a limiting circular aperture, corresponding to beam diameters of 0.7 and $0.35 \mathrm{~cm}$ (areas of 0.38 and $0.096 \mathrm{~cm}^{2}$ ) for eye and skin, respectively. (Note, any small physiological motions, e.g., due to breathing, will have the effect of shifting the illumination point slightly and randomly so that the illuminated area is effectively larger than the spot size calculated from the probe geometry and numerical aperture, here $\sim 0.325 \mathrm{~mm}$ or $0.083 \mathrm{~mm}^{2}$ ). In addition to heat conduction, these factors were taken into account in determining these apertures. ${ }^{54}$ The exposure irradiance $\left(I_{\exp }\right)$ of the optical monitor for each laser is hence calculated as

$I_{\text {exp }}=\frac{P}{A_{\text {lim }}}$,

where $P$ is the measured optical power at the tissue surface.

In the NIR, the permitted illumination irradiance is $\sim 1$ to $2 \mathrm{~mW} / \mathrm{cm}^{2}$ for the eye (point source) and 200 to $375 \mathrm{~mW} / \mathrm{cm}^{2}$ for the skin over total exposure times of 10 to 30,000 s. These total exposure time limits fall within the range of the experiments considered (i.e., with a maximum of $6 \mathrm{~h}$ or 21,600 s). The resulting maximal power per area (MPE) values are summarized in Table 3 for $100 \%$ and $35 \%$ duty cycles. Note that in the studies described here, as in most clinical applications, the highest power laser (i.e., DCS) utilizes a duty cycle $\leq 35 \%$. The DOS system is modulated at $\mathrm{RF}$ frequencies (here, $110 \mathrm{MHz}$ ) and can be considered effectively continuous wave (CW) for the purposes of this calculation; ${ }^{45}$ the DCS laser is CW.

No standards are currently available for the spinal cord. Standards exist for the retina; however, although the retina standards include allowance for the focusing optics in the eye. The neural tissue of the retina is highly specialized for light detection with extremely sensitive photoreceptors, i.e., with structures that strongly absorb light. Since the spinal cord does not contain such photoreceptors, we expect the spinal cord to be less vulnerable to optical damage than the retina. The DCS exposure irradiances we calculated for the present instrumentation were less than the MPE for skin but higher than that specified for the retina, including the $35 \%$ duty cycle (e.g., $\left.0.35 \times I_{\text {skin }}=98 \mathrm{~mW} / \mathrm{cm}^{2}<297 \mathrm{~mW} / \mathrm{cm}^{2}\right)$; DOS irradiances were well below the skin limits and only exceeded eye MPE at $100 \%$ duty cycle (the $0.4 \%$ duty cycle used in this work is below the eye MPE).

\subsection{Neurological Evaluation of Laser Safety}

All animals were evaluated for neurological injury. After the illumination exposure experiment, the fiber-optic probes were removed, and the sheep were permitted to recover. Daily neurological evaluations were conducted on postoperative day 1 and day 2 using the modified Tarlov scale. ${ }^{55}$ The scoring system is described in Table 4. At the end of the $48 \mathrm{~h}$, the sheep were 
Busch et al.: Laser safety in fiber-optic monitoring of spinal cord hemodynamics...

Table 3 MPE and calculated Irradiance $\left(I_{\text {skin/eye }}\right)$ for the relevant wavelengths at $100 \%$ and experimental duty cycle $(d c)$.

\begin{tabular}{|c|c|c|c|c|c|c|c|}
\hline & $\lambda(\mathrm{nm})$ & $\begin{array}{c}\mathrm{MPE}_{\text {eye }} \\
\left(\mathrm{mW} / \mathrm{cm}^{2}\right) \\
\end{array}$ & $\begin{array}{c}1 \times l_{\text {eye }} \\
\left(\mathrm{mW} / \mathrm{cm}^{2}\right) \\
\end{array}$ & $\begin{array}{c}d c \times l_{\text {eye }} \\
\left(\mathrm{mW} / \mathrm{cm}^{2}\right)\end{array}$ & $\begin{array}{c}\mathrm{MPE}_{\text {skin }} \\
\left(\mathrm{mW} / \mathrm{cm}^{2}\right) \\
\end{array}$ & $\begin{array}{c}1 \times I_{\text {skin }} \\
\left(\mathrm{mWW} / \mathrm{cm}^{2}\right) \\
\end{array}$ & $\begin{array}{r}d c \times I_{\text {skin }} \\
\left(\mathrm{mW} / \mathrm{cm}^{2}\right) \\
\end{array}$ \\
\hline DOS & 686 & 1.0 & 7.0 & 0.03 & 200 & 28 & 0.11 \\
\hline DOS & 751 & 1.3 & 10.4 & 0.04 & 253 & 42 & 0.17 \\
\hline DOS & 831 & 1.8 & 10.4 & 0.04 & 366 & 42 & 0.17 \\
\hline DCS & 786 & 1.5 & 70.2 & 24.6 & 297 & 280 & 98 \\
\hline
\end{tabular}

Note: Experimental duty cycles: $d c=0.35$ (DCS) and $d c=0.004$ (DOS). All DOS measurement intensities are well below the MPE for skin but are above MPE for eye at $100 \%$ duty cycle. The DCS irradiance is near MPE for skin at a $100 \%$ duty cycle, but much lower than the MPE at the $\leq 35 \%$ duty cycle utilized here. We include calculations for the eye as this is the only neural tissue with defined ANSI standards, although it is more susceptible to damage, given its specialized photoreceptors.

Table 4 The modified Tarlov scale was used for the neurological scoring and evaluation of sheep for a period of $48 \mathrm{~h}$ after surgery.

\begin{tabular}{ll}
\hline Scale & \multicolumn{1}{c}{ Motor function } \\
\hline 0 & No hind limb movement \\
1 & $\begin{array}{l}\text { Hind limb motion without gravity, } \\
\text { barely perceptible }\end{array}$ \\
2 & $\begin{array}{l}\text { Hind limb motion against gravity but } \\
\text { unable to support weight }\end{array}$ \\
3 & Able to stand, crawl with assistance \\
4 & Able to walk with assistance \\
5 & Normal \\
\hline
\end{tabular}

euthanized and the spinal cord was resected for histological analysis as described in Sec. 2.6.

\subsection{Histological Assessment}

To determine histologically whether there was evidence of surface injury related to laser heating immediately following euthanasia, the spinal cords were excised and fixed in $10 \%$ buffered formalin followed by processing for paraffin embedment. Spinal cord levels (axial regions) excised included the following regions: (1) radiographically documented level of probe tip, (2) one level (vertebra) above, and (3) one level below final probe tip position. Paraffin-embedded tissue sections cut at $5-\mu \mathrm{m}$ thickness were stained with hematoxylin and eosin (H\&E) with three sections produced per spinal cord level. A neuropathologist examined H\&E slides under light microscopy in a blinded fashion. Neuronal injury was evaluated using a semiquantitative scoring system for ischemic neuronal change in ventral gray motor neurons adapted from Celik et al.: ${ }^{56}$ no injury, mild injury ( $<5 \%$ motor neurons with ischemic change), moderate injury (5\% to $20 \%$ motor neurons with ischemic change), and severe injury ( $>20 \%$ motor neurons with ischemic change). H\&E slides were also reviewed to note presence or absence of changes from surface thermal injury.

\subsection{Ex vivo Evaluation of Tissue Heating}

Employing a local thermometry approach, we performed ex vivo experiments to calculate the tissue heating, if any, due to prolonged laser exposure. Spinal cord sections from two sheep were resected after euthanasia. In a model of the worst-case situation, we directly embedded our fiber-optic probe, along with a thermometry probe (Oxford Optronics, United Kingdom), coaxially into the core of freshly resected, $\sim 3 \mathrm{~cm}$, spinal cord sections. The spinal cord tissue, along with the two probes, was suspended in a normal saline bath maintained at $37^{\circ} \mathrm{C}$ to replicate in vivo conditions and the DOS/DCS sources illuminated at a duty cycle of $35 \%$ (i.e., the same settings as utilized for the in vivo measurements). The local tissue temperature was then monitored over $4 \mathrm{~h}$, with recordings made every hour.

\section{Results}

No neurological damage to the spinal cord was observed in either daily neurological evaluation or in spinal cord histopathology.

\subsection{Neurological Evaluation of Laser Safety}

Sequential neurological monitoring of 11 sheep exposed to the laser source for 4 or $6 \mathrm{~h}$ revealed no signs of paresis or paralysis. The modified Tarlov score was 5 in all cases, which indicated that the sheep were normal and showed no signs of any neurological sequelae.

\subsection{Histological Assessment}

Histological analysis was performed on 33 spinal cord tissue samples resected from 11 sheep ( 3 slides per sheep representing the levels above, below, and at the site where the tissue was exposed to the laser source). We found no evidence of injury in any of the 33 sections examined.

Figure 2 shows a representative set of H\&E-stained spinal cord tissue section observed under the light microscope at $2 \times, 20 \times$, and $40 \times$ magnifications with normal neurons and no signs of neuronal ischemic injury associated with thermal heating due to exposure to the laser source.

\subsection{Ex Vivo Evaluation of Tissue Heating}

There was no increase in temperature over $4 \mathrm{~h}$ in both of the resected samples of spinal cord tissue (Table 5). Note that 

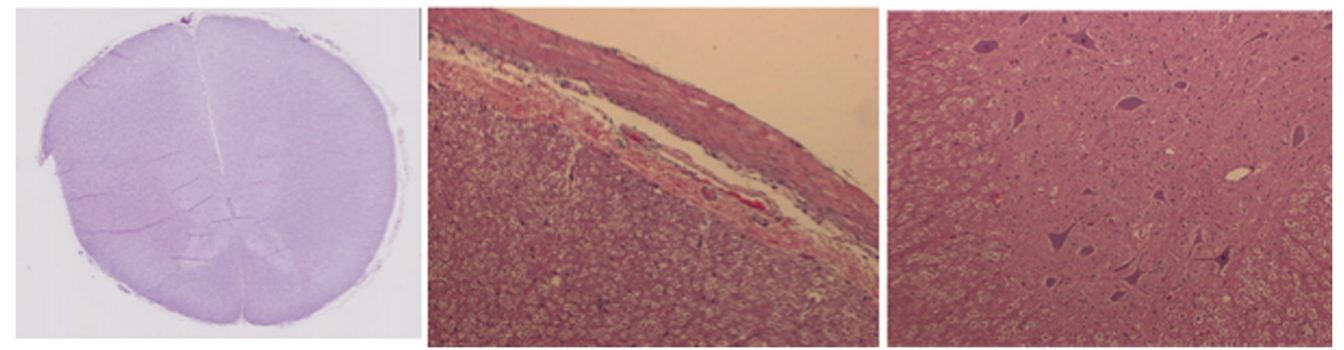

Fig. 2 Spinal cord tissue stained with H\&E showing neurons with normal morphology. Representative images from a single sheep showing the whole cross section of the spinal cord (left, $2 \times$ magnification), close up showing normal spinal cord tissue surface (40x magnification), and an image showing normal neuronal morphology in the irradiated tissue (20x magnification).

Table 5 Temperature monitoring of resected spinal cord. No relevant change in temperature of the two resected spinal cord tissue samples was observed after being directly exposed to the laser source of the fiber-optic probe for $4 \mathrm{~h}$.

Temperatures $\left({ }^{\circ} \mathrm{C}\right)$

\begin{tabular}{lcc}
\hline \multirow{2}{*}{ Time $(\mathrm{h})$} & \multicolumn{2}{c}{ Sheep \# } \\
\cline { 2 - 3 } 0 & 36.8 & \multicolumn{1}{c}{37.1} \\
\hline 1 & 36.8 & 37.0 \\
2 & 37.0 & 37.0 \\
3 & 36.9 & 36.9 \\
4 & 37.1 & 36.9 \\
Mean \pm SD & $36.9 \pm 0.1$ & $37.0 \pm 0.1$ \\
\hline
\end{tabular}

this experiment was designed to mimic a "worst-case" scenario, with zero perfusion and primarily conductive heat transfer from the well-enclosed spinal cavity. The average and standard deviations of temperature measurements in the two sheep were $36.9^{\circ} \mathrm{C} \pm 0.13^{\circ} \mathrm{C}$ and $37.0^{\circ} \mathrm{C} \pm 0.08^{\circ} \mathrm{C}$.

\section{Discussion}

In this study, we estimated and evaluated the safety of laser light illumination for continuous diffuse optical hemodynamics monitoring of the spinal cord in sheep, utilizing a probe placed within the epidural space. First, we estimated the light irradiance on the spinal cord due to illumination from DOS/DCS lasers, operating at a specified duty cycle. At duty cycles that are typical for long-term continuous monitoring of tissue, these tissue irradiances were less than the MPEs defined by ANSI (Z136.1) for skin (no such limits are given for spinal tissue). Furthermore, we found that exposure of the spinal cord to light did not cause observable histological or functional neurological damage. The findings of this multifaceted assessment (histopathology, neurological evaluations, and ex vivo measurements) demonstrate the safety of the DOS/DCS technique for spinal cord monitoring, which may ultimately fill a critical gap in neurocritical care monitoring.

For the wavelength range and continuous illumination (i.e., lasers are not pulsed with high peak powers) considered in this study, the risk to tissue from optical illumination is expected to be exclusively thermal. ${ }^{45}$ Thermal risks include coagulation, protein denaturation, and cell death; indeed these are frequently the goal of photothermal therapies. ${ }^{57}$ However, these effects are all dependent upon input optical power and irradiance, as well as the thermal conductivity of tissue.

The neural tissue of the spine is protected by the dura $(\sim 0.3-\mathrm{mm} \text { thick })^{58}$-tissue that is somewhat analogous to skin in considerations of laser safety. At optical wavelengths, this dura layer scatters light, which has the effect of diffusing light power over a broader area than the spot emanating from the optical fibers prior to illumination of the neural tissue. Recent computational work examined heating in the various layers of the head (also with a superficial skin layer) due to illumination at $800 \mathrm{~nm}$. The computational results $(10 \mathrm{~mW}$ at $100 \%$ duty cycle) that most closely resemble the work described (i.e., $27 \mathrm{~mW}$ at $35 \%$ duty cycle) suggest that laser illumination could potentially induce a highly localized $1.5^{\circ} \mathrm{C}$ increase in temperature. ${ }^{59}$ However, our experimental results using thermometry did not demonstrate such a change in the spinal cord with the illumination parameters described above, despite our thermal experiment occurring in a worst-case scenario of zero blood flow (excised tissue). We note that the catheter wall in our probe is $\sim 0.4-\mathrm{mm}$ thick and the beam exiting the fiber-optic is diverging ( $0.22 \mathrm{NA})$, both of which serves to minimize localized heating effects. In our ex vivo experiment, the introduction of the thermal probe could possibly distort/ impact the distribution of light intensity in the spinal cord tissues. However, these light intensity distortions would more likely cause an increase (e.g., due to absorption) in deposited radiant energy, thus enhancing, rather than reducing, any thermal effects. In vivo, the high blood flow $(\approx 30$ to $60 \mathrm{ml} / 100 \mathrm{~g} / \mathrm{min})^{60,61}$ of the spinal cord would also help disperse heat. Furthermore, cerebrospinal fluid (CSF) has a higher heat conduction and capacity than air (to which skin is typically exposed), increasing thermal dissipation, and CSF flow likely further reduces localized heating of the spinal cord.

We did not observe cell damage in histopathological analysis following exposure of the spinal cord to $98 \mathrm{~mW} / \mathrm{cm}^{2}$ at a $35 \%$ duty cycle (i.e., $\sim 1 / 3$ of the skin MPE and $\sim 18 \times$ the ocular MPE). Furthermore, in a worst-case scenario of zero perfusion in an ex vivo spinal column in a thermal bath (mimicking the remainder of the body), local tissue temperature was constant within $1 / 4^{\circ} \mathrm{C}$. We note that power deposition is directly proportional to the optical duty cycle, which suggests that concerns about the level of optical power deposition can be mitigated by appropriately reducing the illumination duty cycle to the minimum required to achieve the necessary temporal measurement 
resolution. Furthermore, these DCS data were not collected at the minimum necessary signal-to-noise ratio for the DCS technique, i.e., additional reductions in the deposited optical power are possible simply by attenuating the lasers.

It is important to distinguish the work presented here from previous reports involving focused and pulsed lasers or highspeed scanning of the illumination point. Focused and pulsed lasers deliver much higher intensities at similar average powers (e.g., 25-s exposure, average power $13 \mathrm{~mW}, \lambda=704,880 \mathrm{~nm}$, 2.5-ps pulses, and peak intensities $\left.\sim 2 \times 10^{11} \mathrm{~W} / \mathrm{cm}^{2}\right){ }^{62}$ Although the average power is roughly half of that described in this work, the peak intensity is many orders of magnitude higher, which may cause the formation of plasmas or cavitation; the unfocused, diverging, continuous illumination utilized here is incapable of producing plasmas, etc. As per high-speed scanning, although there are numerous reports of scanning imaging techniques utilized on the spine, the illumination at a single point is brief. In these studies, the spine can be illuminated at higher powers (e.g., during coherent anti-stokes Raman scattering microscopy), but the point of focus is scanned rapidly $(<1 \mathrm{~s})$. Thus, although the power densities are similar (with identical limiting apertures, 50 to $100 \mathrm{~mW}$ versus $27 \mathrm{~mW}$, Table 2), rapid scanning permits thermal diffusion and lessens the probability for injury compared with the continuous stationary illumination utilized in this paper.

Several potential limitations exist for this study. These limitations primarily arise from probe-tissue geometry and positioning. A rotationally misaligned probe, or an off-center probe, could result in a lower power deposition than a probe that is well centered with lasers directed perpendicular to the spinal cord; due to space constraints, we did not emplace continuous optical dosimetry sensors. Similarly, the probe may be inadvertently placed below the dura into the subdural space instead of the epidural space; however, such an arrangement could only enhance the power deposition into the neuronal tissues. It may be useful to conduct future testing with purposeful placement of the probe below the dura to assure laser safety in a worst-case scenario. Additionally, measurements of highly localized $\left(\sim 1 \mathrm{~mm}^{3}\right)$ injury causing subtle changes in spinal function are beyond the scope of this work; this will require precise coregistration of illumination, histology, and functional testing. Modeling of light transport and simulations of heat deposition in the spinal cord and skin will assist interpretation of future work. We have studied the effect of laser illumination on a healthy, well-perfused spinal cord; an ischemic cord may be more susceptible to injury. Potential future studies include examination of spinal cord susceptibility to laser damage in the perfused and ischemic state and regional variation in susceptibility to damage. We note that we did not explicitly examine the impact of long-term (days to weeks) exposure; long-term damage is unlikely since we did not observe any physiological or structural changes due to light illumination, but subtle alterations in function cannot be ruled out. This lack of data on longterm chronic exposures is a well-known problem in the field, as noted in international standards. ${ }^{54}$ Moreover, we did not attempt to define an upper limit of safe tissue exposure to laser illumination. This more comprehensive study is beyond the current limitations of our instrumentation, i.e., our monitoring system was designed to be incapable of exceeding the ANSI skin limits, which have a significant safety margin prior to tissue injury. We note that, despite this restriction, the signal-to-noise ratio of our current system is quite high, permitting measurement of blood flow in likely clinical scenarios and fiber geometries. Many clinical scenarios require relatively low time resolution in measurement of blood flow ( $30 \mathrm{~s})$, permitting significant reduction in the optical thermal input to tissue. Safety calculations for the eye must take into account the integral focusing optics; the power density on the retina may be considerably greater than that on the cornea. The spinal cord does not have such focusing optics, and thus, the direct comparison of these standards is problematic. We include the calculations in this paper to place the spinal cord data demonstrated here in context with the retinal limits as both a familiar value to many in the field and the only neural tissue with established exposure limits. We note that, without establishing the upper limit for safe exposure, we cannot determine the ratio of our current exposure to the "true" MPE of the spinal cord. Finally, this study utilized a large animal model, closely mimicking human geometry. Like many large animal studies, logistical constraints limited the total number of animals studied; additional work will need to follow this initial study prior to translation into humans.

In conclusion, based on sheep studies, diffuse optical measurements provide a minimally invasive, nonionizing, bedside monitor of spinal cord hemodynamics at optical powers that do not cause observable neurological injury. These tools, therefore, should be adequate for monitoring the spinal cord during procedures, which may cause ischemia (e.g., stent placement for aortic aneurysm), or even during spinal cord decompression.

\section{Disclosures}

Dr. Floyd and Dr. Yodh's conflicts include partial ownership of pending patents directly related to this work: WO 2013/090658 $\mathrm{Al}$ and PCT/US2012/069626. Dr. Yodh has partial ownership of two patents relevant to this work (United States patents $8,082,015$ and 6,076,010). Dr. Busch, Dr. Parthasarathy, and Dr. Yodh have partial ownership of two pending patent applications relevant to this work (PCT/US2015/017286 and PCT/ US2015/017277). No author currently receives royalties or payments from these patents. Dr. Floyd is the president and CEO of NFOSYS, Inc., a startup company that may, in the future, be involved with producing and selling this technology.

\section{Acknowledgments}

This work was primarily funded by the Craig H. Neilsen Foundation Senior Research Grant (T.F.F., A.K., and A.G.Y.). The authors acknowledge contributing partial support from the National Institutes of Health (Nos. P41-EB015893 and R01-NS060653, A.G.Y. and A.B.P.), and we thank Dr. David Sliney, Dr. Stefan Carp, and Valerie Perez for their valuable discussions.

\section{References}

1. D. L. Reames et al., "Complications in the surgical treatment of 19, 360 cases of pediatric scoliosis: a review of the Scoliosis Research Society Morbidity and Mortality Database," Spine (Phila Pa 1976) 36(18), 1484-1491 (2011).

2. C. G. Patil et al., "Complications and outcomes after spinal cord tumor resection in the United States from 1993 to 2002," Spinal Cord 46(5), 375-379 (2008).

3. D. A. Becker et al., "Predictors of outcome in patients with spinal cord ischemia after open aortic repair," Neurocrit. Care 18(1), 70-74 (2013).

4. M. F. Conrad et al., "Thoracoabdominal aneurysm repair: a 20-year perspective," Ann. Thorac. Surg. 83(2), S856-S861; discussion S890S852 (2007). 
5. A. S. Kogler et al., "Fiber-optic monitoring of spinal cord hemodynamics in experimental aortic occlusion," Anesthesiology 123(6), 13621373 (2015).

6. R. C. Mesquita et al., "Optical monitoring and detection of spinal cord ischemia," PLoS One 8(12), e83370 (2013).

7. P. Costa et al., "Somatosensory- and motor-evoked potential monitoring during spine and spinal cord surgery," Spinal Cord 45(1), 86-91 (2007).

8. V. Deletis and F. Sala, "Intraoperative neurophysiological monitoring of the spinal cord during spinal cord and spine surgery: a review focus on the corticospinal tracts," Clin. Neurophysiol. 119(2), 248-264 (2008).

9. S. Deiner, "Highlights of anesthetic considerations for intraoperative neuromonitoring," Semin. Cardiothorac. Vasc. Anesth. 14(1), 51-53 (2010).

10. M. Mahmoud et al., "Susceptibility of transcranial electric motorevoked potentials to varying targeted blood levels of dexmedetomidine during spine surgery," Anesthesiology 112(6), 1364-1373 (2010).

11. A. C. Wang et al., "Impact of anesthesia on transcranial electric motor evoked potential monitoring during spine surgery: a review of the literature," Neurosurg. Focus 27(4), E7 (2009).

12. R. P. Lesser et al., "Postoperative neurological deficits may occur despite unchanged intraoperative somatosensory evoked potentials," Ann. Neurol. 19(1), 22-25 (1986).

13. J. Y. Hong et al., "False negative and positive motor evoked potentials in one patient: is single motor evoked potential monitoring reliable method? A case report and literature review,' Spine (Phila Pa 1976) 35(18), E912-E916 (2010).

14. C. ter Wolbeek et al., "Value and pitfalls of neurophysiological monitoring in thoracic and thoracoabdominal aortic replacement and endovascular repair," Thorac. Cardiovasc. Surg. 58(5), 260-264 (2010).

15. C. D. Etz et al., "Spinal cord blood flow and ischemic injury after experimental sacrifice of thoracic and abdominal segmental arteries," Eur. J. Cardiothorac. Surg. 33(6), 1030-1038 (2008).

16. J. Lips et al., "Delayed detection of motor pathway dysfunction after selective reduction of thoracic spinal cord blood flow in pigs," J. Thorac. Cardiovasc. Surg. 123(3), 531-538 (2002).

17. M. R. Nuwer et al., "Evidence-based guideline update: intraoperative spinal monitoring with somatosensory and transcranial electrical motor evoked potentials: report of the Therapeutics and Technology Assessment Subcommittee of the American Academy of Neurology and the American Clinical Neurophysiology Society," Neurology 78(8), 585-589 (2012).

18. I. Phang et al., "Safety profile and probe placement accuracy of intraspinal pressure monitoring for traumatic spinal cord injury: injured spinal cord pressure evaluation study," J. Neurosurg. Spine 25(3), 398-405 (2016).

19. J. W. Squair et al., "Spinal cord perfusion pressure predicts neurologic recovery in acute spinal cord injury," Neurology 89(16), 1660-1667 (2017).

20. V. Jain et al., "Cerebral oxygen metabolism in neonates with congenital heart disease quantified by MRI and optics," J. Cereb. Blood Flow Metab. 34(3), 380-388 (2014).

21. S. A. Carp et al., "Validation of diffuse correlation spectroscopy measurements of rodent cerebral blood flow with simultaneous arterial spin labeling MRI; towards MRI-optical continuous cerebral metabolic monitoring," Biomed. Opt. Express 1(2), 553-565 (2010).

22. E. M. Buckley et al., "Validation of diffuse correlation spectroscopic measurement of cerebral blood flow using phase-encoded velocity mapping magnetic resonance imaging," J. Biomed. Opt. 17(3), 037007 (2012).

23. G. Q. Yu et al., "Validation of diffuse correlation spectroscopy for muscle blood flow with concurrent arterial spin labeled perfusion MRI," Opt. Express 15(3), 1064-1075 (2007).

24. T. Durduran et al., "Optical measurement of cerebral hemodynamics and oxygen metabolism in neonates with congenital heart defects," J. Biomed. Opt. 15(3), 037004 (2010).

25. M. N. Kim et al., "Validation of diffuse correlation spectroscopy against xenon CTCBF in humans after traumatic brain injury or subarachnoid hemorrhage," in Neurocritical Care Society Annual Meeting, Miami, Florida (2008).

26. D. R. Busch, "A collection of papers on applications of diffuse correlation spectroscopy (DCS)," 2017, https://www.physics.upenn.edu/ yodhlab/dcs/ (01 August 2017).
27. T. Durduran et al., "Diffuse optics for tissue monitoring and tomography," Rep. Prog. Phys. 73(7), 076701 (2010).

28. D. A. Boas and A. G. Yodh, "Spatially varying dynamical properties of turbid media probed with diffusing temporal light correlation," J. Opt. Soc. Am. A 14(1), 192-215 (1997).

29. E. M. Buckley et al., "Diffuse correlation spectroscopy for measurement of cerebral blood flow: future prospects," Neurophotonics 1(1), 011009 (2014).

30. J. Selb et al., "Sensitivity of near-infrared spectroscopy and diffuse correlation spectroscopy to brain hemodynamics: simulations and experimental findings during hypercapnia," Neurophotonics 1(1), 015005 (2014).

31. M. Diop et al., "Calibration of diffuse correlation spectroscopy with a time-resolved near-infrared technique to yield absolute cerebral blood flow measurements," Biomed. Opt. Express 2(7), 2068-2081 (2011).

32. E. M. Buckley et al., "Decreased microvascular cerebral blood flow assessed by diffuse correlation spectroscopy after repetitive concussions in mice," J. Cereb. Blood Flow Metab. 35(12), 1995-2000 (2015).

33. C. G. Favilla et al., "Optical bedside monitoring of cerebral blood flow in acute ischemic stroke patients during head-of-bed manipulation," Stroke 45(5), 1269-1274 (2014).

34. D. R. Busch et al., "Cerebral blood flow response to hypercapnia in children with obstructive sleep Apnea syndrome," Sleep 39(1), 209216 (2016).

35. P. Farzam et al., "Noninvasive characterization of the healthy human manubrium using diffuse optical spectroscopies," Physiol. Meas. 35(7), 1469-1491 (2014).

36. S. Han et al., "Non-invasive monitoring of temporal and spatial blood flow during bone graft healing using diffuse correlation spectroscopy," PLoS One 10(12), e0143891 (2015).

37. R. Choe et al., "Optically measured microvascular blood flow contrast of malignant breast tumors," PLoS One 9(6), e99683 (2014).

38. D. R. Busch et al., "Blood flow reduction in breast tissue due to mammographic compression," Acad. Radiol. 21(2), 151-161 (2014).

39. G. Q. Yu et al., "Time-dependent blood flow and oxygenation in human skeletal muscles measured with noninvasive near-infrared diffuse optical spectroscopies," J. Biomed. Opt. 10, 024027 (2005).

40. Y. Shang et al., "Noninvasive optical characterization of muscle blood flow, oxygenation, and metabolism in women with fibromyalgia," Arthritis Res. Ther. 14(6), R236 (2012).

41. C. Huang et al., "Noncontact diffuse optical assessment of blood flow changes in head and neck free tissue transfer flaps," J. Biomed. Opt. 20(7), 075008 (2015).

42. Y. Shang et al., "Cerebral monitoring during carotid endarterectomy using near-infrared diffuse optical spectroscopies and electroencephalogram," Phys. Med. Biol. 56(10), 3015-3032 (2011).

43. D. R. Busch et al., "Continuous cerebral hemodynamic measurement during deep hypothermic circulatory arrest," Biomed. Opt. Express 7(9), 3461-3470 (2016).

44. Laser Institute of America, "American National Standard for the safe use of lasers: ANSI Z136.3-2011," Laser Institute of America, Orlando, Florida (2011).

45. Laser Institute of America, "American National Standard for the safe use of lasers: ANSI Z136.1-2014," Laser Institute of America, Orlando, Florida (2014).

46. D. S. Choy, "Percutaneous laser disc decompression: a 17-year experience," Photomed. Laser Surg. 22(5), 407-410 (2004).

47. S. Kobayashi et al., "A case of nerve root heat injury induced by percutaneous laser disc decompression performed at an outside institution: technical case report," Oper. Neurosurg. 60(Suppl. 2), ONS-E171ONS-E172 (2007).

48. M. S. Moreira et al., "Effect of laser phototherapy on wound healing following cerebral ischemia by cryogenic injury," J. Photochem. Photobiol. B 105(3), 207-215 (2011).

49. S. Veronez et al., "Effects of different fluences of low-level laser therapy in an experimental model of spinal cord injury in rats," Lasers Med. Sci. 32(2), 343-349 (2017).

50. S. R. Sheng et al., "Anatomy of large animal spines and its comparison to the human spine: a systematic review," Eur. Spine J. 19(1), 46-56 (2010).

51. T. Durduran and A. G. Yodh, "Diffuse correlation spectroscopy for non-invasive, micro-vascular cerebral blood flow measurement," Neurolmage 85(Pt. 1), 51-63 (2014). 
52. D. A. Boas, L. E. Campbell, and A. G. Yodh, "Scattering and imaging with diffusing temporal field correlations," Phys. Rev. Lett. 75(9), 18551858 (1995).

53. R. C. Mesquita and A. Yodh, "Diffuse optics: fundamentals and tissue applications," in Proceedings of the International School of Physics "Enrico Fermi," Nano Optics and Atomics: Transport of Light and Mater Waves, R. Kaiser, D. S. Weirsma, and L. Fallini, Eds., IOS Press, Amsterdam (2011).

54. International Commission on Non-Ionizing Radiation Protection, "Protection ICoN-IR. ICNIRP guidelines on limits of exposure to laser radiation of wavelengths between $180 \mathrm{~nm}$ and $1000 \mu \mathrm{m}$," Health Phys. 105(3), 271-295 (2013).

55. P. G. Popovich et al., "A reassessment of a classic neuroprotective combination therapy for spinal cord injured rats: LPS/pregnenolone/indomethacin," Exp. Neurol. 233(2), 677-685 (2012).

56. M. Celik et al., "Erythropoietin prevents motor neuron apoptosis and neurologic disability in experimental spinal cord ischemic injury," Proc. Natl. Acad. Sci. U. S. A. 99(4), 2258-2263 (2002).

57. S. Thomsen, "Pathologic analysis of photothermal and photomechanical effects of laser-tissue interactions," Photochem. Photobiol. 53(6), 825-835 (1991)

58. J. Y. Hong et al., "Analysis of dural sac thickness in human spinecadaver study with confocal infrared laser microscope," Spine J. 11(12), 1121-1127 (2011).

59. M. Nourhashemi, M. Mahmoudzadeh, and F. Wallois, "Thermal impact of near-infrared laser in advanced noninvasive optical brain imaging," Neurophotonics 3(1), 015001 (2016).

60. G. Zoccoli et al., "Spinal cord blood flow changes during the sleepwake cycle in rat," Neurosci. Lett. 163(2), 173-176 (1993).

61. S. H. Johnson, J. M. Kraimer, and G. M. Graeber, "Effects of flunarizine on neurological recovery and spinal cord blood flow in experimental spinal cord ischemia in rabbits," Stroke 24(10), 15471553 (1993).

62. Y. Fu et al., "Characterization of photodamage in coherent antiStokes Raman scattering microscopy," Opt. Express 14(9), 3942-3951 (2006).

David R. Busch is an assistant professor in the Department of Anesthesiology and Pain Management and Department of Neurology and Neurotherapeutics, the University of Texas Southwestern Medical Center. His work focuses on developing noninvasive and minimally invasive optical tools to monitor deep tissues in health and disease, as well as throughout therapy.

Ashwin B. Parthasarathy is an assistant professor of electrical engineering at the University of South Florida, Tampa, Florida. His Translational Optics Imaging and Spectroscopy Lab is focused on the development and application of optical spectroscopy and microscopy techniques for the bedside monitoring and imaging of tissue physiology in human and animal models.

Arjun G. Yodh is a Skinner professor of science in the Department of Physics and Astronomy, the University of Pennsylvania. He also directs the Laboratory for Research on the structure of matter, an interdisciplinary materials institute that hosts the Penn MRSEC. His biomedical research is oriented toward diffuse optical imaging and monitoring, with aims to demonstrate the potential of these tools for functional imaging/monitoring in brain, breast, and muscle, and for monitoring hemodynamic biomarkers during treatment.

Biographies for the other authors are not available. 